\title{
Human Origins in Southern African Palaeo-wetlands? Strong Claims from Weak Evidence
}

Carina M Schlebusch ${ }^{1,2,3, * \#, \text { Liisa Loog }}{ }^{4 *}$, Huw S Groucutt ${ }^{5}$, Turi King ${ }^{6}$, Adam Rutherford ${ }^{7}$, Chiara Barbieri $^{8}$, Guido Barbujani ${ }^{9}$, Lounes Chikhi ${ }^{10}$, Mattias Jakobsson ${ }^{1,2,3}$, Anders Eriksson ${ }^{11}$, Andrea Manica $^{12}$, Sarah A Tishkoff ${ }^{13}$, Eleanor ML Scerri ${ }^{14}$, Aylwyn Scally ${ }^{4}$, Chris Brierley ${ }^{15}$, Mark G Thomas ${ }^{7}$ \#

*Equal contribution

\#Correspondence: carina.schlebusch@ebc.uu.se and m.thomas@ucl.ac.uk

1 Human Evolution, Department of Organismal Biology, Uppsala University, Sweden

2 Palaeo-Research Institute, University of Johannesburg, South Africa

3 SciLifeLab Uppsala, Sweden

4 Department of Genetics, University of Cambridge, UK

5 Extreme Events Research Group, Max Planck Institute for Chemical Ecology, Jena, Germany.

6 Department of Genetics and Genome Biology \& School of Archaeology and Ancient History, University of Leicester, UK

7 Department of Genetics, Evolution and Environment, University College London, UK

8 Department of Evolutionary Biology and Environmental Studies, University of Zurich, Zurich, Switzerland

9 Department of Life Sciences and Biotechnology, University of Ferrara, Italy

10 Evolution \& Diversité Biologique lab, CNRS, Univ. Toulouse 3, France - Instituto Gulbenkian de Ciência, Oeiras, Portugal

11 Center for Genomics, Evolution and Medicine (cGEM), University of Tartu, Estonia

12 Department of Zoology, University of Cambridge, UK

13 Departments of Genetics and Biology, University of Pennsylvania, Philadelphia, PA USA

14 Pan-African Evolution Research Group, Max Planck Institute for the Science of Human History, Jena, Germany

15 Department of Geography, University College London, UK

Keywords: human origins, mitochondrial DNA; Africa; human evolution 
Chan and colleagues in their paper titled "Human origins in a southern African palaeo-wetland and first migrations" (https://www.nature.com/articles/s41586-019-1714-1) report 198 novel whole mitochondrial DNA (mtDNA) sequences and infer that 'anatomically modern humans' originated in the Makgadikgadi-Okavango palaeo-wetland of southern Africa around 200 thousand years ago. This claim relies on weakly informative data. In addition to flawed logic and questionable assumptions, the authors surprisingly disregard recent evidence and debate on human origins in Africa $^{1-4}$. As a result, the emphatic and high profile conclusions of the paper are unjustified.

\section{Weakly informative data}

Chan et al. sampled 198 Southern African individuals, sequenced the full mtDNA genome and merged their data with 1000 other published mtDNA genomes, mostly from southern Africa. They then proceed by inferring the phylogenetic structure of the mtDNA genealogy, estimating the ages of various branches on that tree (with a particular focus on branches found in southern Africa), and considering their modern geographic distribution. Mitochondrial DNA can be useful for addressing certain population history questions ${ }^{5-7}$, and it is good to focus on under-sampled populations, but the phylogenetic tree at any single non-recombining locus (such as mtDNA) is only one random outcome of the genealogical process and is weakly constrained by population history. In practice, this means it contains useful but limited information about that population history. In particular, information content reduces rapidly as we look further back in time. At the time when all mtDNA lineages coalesce to their most recent common ancestor - the part of the tree where Chan and colleagues focus their analysis - the mtDNA lineage represent only one of the thousands of ancestors that present-day humans have at that time, and therefore provides little or no information to distinguish between plausible models of human population history.

If we consider another well-resolved single locus phylogenetic tree - that of the Y chromosome - a different phylogeographic structure is apparent ${ }^{8}$. Modern population genetic approaches take advantage of hundreds of thousands of independent gene trees, gaining several orders of magnitude in statistical power ${ }^{9}$. While each tree is only weakly constrained by demographic history, by combining many trees with probabilistic approaches it is possible to quantify the likelihood of different demographic scenarios. However, in the absence of constraining ancient DNA data from the periods in question, precise inferences on the location of origin of Homo sapiens require strong and likely unjustified assumptions about human mobility in the intervening period, and so must be treated with caution. Genome-wide studies of contemporary and Holocene African populations indicate complex demography, including deep structure and varying migration rates over time ${ }^{4,10-18}$. However, despite the far greater information content, no genome-wide study has been able to justify such geographic and temporal precision for modern human origins as that of Chan and colleagues.

\section{Lineages are not populations}

While the methods used to estimate the mtDNA tree and its branch dates are relatively uncontroversial, it is important to note that there is no natural level at which those branches should be assigned haplogroup status, and no special meaning for the age of any arbitrarily defined haplogroup. Chan and colleagues arbitrarily choose lineages and treat them as representing real populations of prehistoric humans. Lineages in a gene tree correspond to lines of parent-offspring 
descent, but demonstrably do not correspond to populations today, and there is little reason to assume that they did in the past. There is also little reason to assume that branch split dates in the tree correspond directly to population-level splitting events. Furthermore, Chan and colleagues apply Bayesian Skyline analysis to their arbitrarily selected lineages to infer ancestral population size change through time. This imposes a sampling bias that breaks key assumptions of Bayesian Skyline analysis (i.e. the data are from a lineage sample rather than from an unstructured population sample), invalidating the reconstructions of population sizes through time ${ }^{19}$.

\section{Populations are not static}

A key issue with inferring geographic origins from contemporary genetic data is the extent to which population history is a migrational palimpsest in the intervening period. Chan and colleagues' claims rest on the implicit assumption that the locations of present-day individuals represent those of past populations. This assumption of static populations over a period of more than 100,000 years is problematic in the light of well described (by both archaeological and ancient DNA studies) long and short range migrations, population contractions, expansions and replacements - not only in Eurasia, the Americas and Oceania, but also in Africa (e.g. ${ }^{12-14,16}$ ). The assumption of static populations would need to be supported by archaeological, fossil, and ideally ancient DNA evidence; to date no such evidence has been presented for such a deep time period anywhere in Africa. On the contrary, studies of raw material transport demonstrate high levels of mobility among Pleistocene hunter-gatherers that are at odds with the notion of isolated populations over a period of more than 100,000 years (e.g. ${ }^{20,21}$ ). This issue remains regardless of whether single locus (e.g. mtDNA) data or more strongly informative multi-locus genomic data is considered.

\section{Palaeoanthropology}

Chan and colleagues conclude that 'anatomically modern humans' evolved in southern Africa around 200,000 years ago. However, hominin fossil data from across Africa indicate the presence of morphology characteristic to Homo sapiens more than 300 thousand years ago at the opposite end of the continent ${ }^{22}$. The evolution of Homo sapiens morphology is characterised by a mosaic of different derived and primitive features across time and space, and these data do not indicate a single origin point $^{1,23,24}$. Chan and colleagues use archaeological evidence for 'modern human behaviour' from southern Africa between 60 and 100 ka to corroborate their claims of human origins in that region ${ }^{25}$. However, evidence of complex culture is found in other African regions around this period (e.g. ${ }^{20}$, ${ }^{21}$ ). Together, current palaeoanthropological data do not support claims that one single area of Africa was the 'homeland' of Homo sapiens.

\section{Climate reconstructions}

Using climate reconstructions to contextualise inferences based on genetic data is a commendable aim. However, while Chan and colleagues argue that wetlands were a stable habitat, they give no evidence that wetlands provide ecologies that are well-suited to ancient humans, and do not consider palaeo-wetlands elsewhere in Africa ${ }^{26}$. Without addressing these issues it is difficult to see how any type of climate reconstruction can provide a test of their origins model. Other ecologies, such as 
grasslands, savannahs and Mediterranean biomes were also inhabited by $H$. sapiens for long periods of time, and were therefore clearly suitable for long term Homo sapiens occupation ${ }^{1,22}$. A more plausible model is that strong variations in climate on the seasonal/annual timescales, but without potentially deadly multi-year droughts/floods, would be more optimal for human occupation. The climate model that Chan et al employ would not capture those features well. Relationships between climate and human demography are best explored through spatially explicit modelling ${ }^{27,28}$ rather than post-hoc explanations which ignore other data from across Africa ${ }^{29}$.

\section{Concluding remarks}

Although admittedly common in human evolutionary studies, a particularly troubling aspect of Chan and colleagues' study is that it ignores the central pillar of modern science: hypothesis testing. The authors make no attempt to quantify how well their data can be explained either by simpler models of population history than the one they claim, or by equally complex but dramatically different models, such as those involving an origin of Homo sapiens elsewhere in Africa, or indeed, in multiple locations throughout Africa.

100 years ago, in the first study using biomarkers, Hirschfeld and Hirschfeld (1919) typed different populations from around the world at a single genetic locus (ABO blood groups) ${ }^{30}$. While the study was revolutionary, the authors concluded that there were two ancestral human 'races', corresponding to the A and B blood groups, with two different geographical origins that subsequently mixed globally. Subsequent developments in population genetics, genomics, archaeology and paleoanthropology have revealed a very different and more complex picture. But the dangers of basing conclusions on limited data and flawed assumptions remain just as relevant today. Presentation of such studies, with predictably high-profile attention and simple yet questionable results, does not serve the science of human origins, nor its broader dissemination. Instead, prominent media coverage frequently presents the results uncritically, and so contributes misinformation about our evolution.

\section{References}

1. Scerri, E. M. L. et al. Did Our Species Evolve in Subdivided Populations across Africa, and Why Does It Matter? Trends Ecol Evol 33, 582-594 (2018).

2. Scerri, E. M. L., Chikhi, L. \& Thomas, M. G. Beyond multiregional and simple out-of-Africa models of human evolution. Nat Ecol Evol 3, 1370-1372 (2019).

3. Henn, B. M., Steele, T. E. \& Weaver, T. D. Clarifying distinct models of modern human origins in Africa. Curr Opin Genet Dev 53, 148-156 (2018).

4. Schlebusch, C. M. \& Jakobsson, M. Tales of Human Migration, Admixture, and Selection in Africa. Annu Rev Genomics Hum Genet 19, 405-428 (2018).

5. Barbieri, C. et al. Unraveling the complex maternal history of Southern African Khoisan populations. Am J Phys Anthropol 153, 435-48 (2014).

6. Barbieri, C. et al. Ancient substructure in early mtDNA lineages of southern Africa. Am J Hum Genet 92, 285-92 (2013).

7. Karmin, M. et al. A recent bottleneck of $Y$ chromosome diversity coincides with a global change in culture. Genome Res 25, 459-66 (2015).

8. Mendez, F. L. et al. An African American paternal lineage adds an extremely ancient root to the human Y chromosome phylogenetic tree. Am J Hum Genet 92, 454-9 (2013). 
9. Kelleher, J. et al. Inferring whole-genome histories in large population datasets. Nat Genet 51, 13301338 (2019).

10. Tishkoff, S. A. et al. The genetic structure and history of Africans and African Americans. Science 324, 1035-44 (2009).

11. Schlebusch, C. M. et al. Genomic Variation in Seven Khoe-San Groups Reveals Adaptation and Complex African History. Science 338, 374-379 (2012).

12. Schlebusch, C. M. et al. Ancient genomes from southern Africa pushes modern human divergence beyond 260,000 years ago. bioRxiv (2017).

13. Pickrell, J. K. et al. The genetic prehistory of southern Africa. Nat Commun 3, 1143 (2012).

14. Skoglund, P. et al. Reconstructing Prehistoric African Population Structure. Cell 171, 59-71 e21 (2017).

15. Mazet, O., Rodriguez, W., Grusea, S., Boitard, S. \& Chikhi, L. On the importance of being structured: instantaneous coalescence rates and human evolution--lessons for ancestral population size inference? Heredity (Edinb) 116, 362-71 (2016).

16. Bergström, A. et al. Insights into human genetic variation and population history from 929 diverse genomes. bioRxiv Jan 1:674986. (2019).

17. Henn, B. M. et al. Hunter-gatherer genomic diversity suggests a southern African origin for modern humans. Proc Natl Acad Sci U S A 108, 5154-62 (2011).

18. Fan, S. et al. African evolutionary history inferred from whole genome sequence data of 44 indigenous African populations. Genome Biol 20, 82 (2019).

19. Heller, R., Chikhi, L. \& Siegismund, H. R. The confounding effect of population structure on Bayesian skyline plot inferences of demographic history. PLoS One 8, e62992 (2013).

20. Scerri, E. M. L. The North African Middle Stone Age and its place in recent human evolution. Evol Anthropol 26, 119-135 (2017).

21. Brooks, A. S. et al. Long-distance stone transport and pigment use in the earliest Middle Stone Age. Science 360, 90-94 (2018).

22. Hublin, J. J. et al. New fossils from Jebel Irhoud, Morocco and the pan-African origin of Homo sapiens. Nature 546, 289-292 (2017).

23. Gunz, P. et al. Early modern human diversity suggests subdivided population structure and a complex out-of-Africa scenario. Proc Natl Acad Sci U S A 106, 6094-8 (2009).

24. Stringer, C. The origin and evolution of Homo sapiens. Philos Trans R Soc Lond B Biol Sci 371 (2016).

25. Henshilwood, C. S. The Still Bay and Howiesons Poort: 'Palaeolithic' techno-traditions in southern Africa. J World Pre-hist 25 205-237 (2012).

26. Wrangham, R., Cheney, D., Seyfarth, R. \& Sarmiento, E. Shallow-water habitats as sources of fallback foods for hominins. Am J Phys Anthropol 140, 630-42 (2009).

27. Timmermann, A. \& Friedrich, T. Late Pleistocene climate drivers of early human migration. Nature 538, 92-95 (2016).

28. Eriksson, A. \& Manica, A. Effect of ancient population structure on the degree of polymorphism shared between modern human populations and ancient hominins. Proc Natl Acad Sci U S A 109, 13956-60 (2012).

29. Blome, M. W., Cohen, A. S., Tryon, C. A., Brooks, A. S. \& Russell, J. The environmental context for the origins of modern human diversity: a synthesis of regional variability in African climate 150,00030,000 years ago. J Hum Evol 62, 563-92 (2012).

30. Hirschfeld, L. \& Hirschfeld, H. Serological Differences between the Blood of Different Races. The Lancet 194, 675-679 (1919). 